\title{
Prevalence of Anti-deamidated Gliadin Peptide Antibodies in Asian Patients With Irritable Bowel Syndrome
}

\author{
Wei Lu, ${ }^{1}$ Kok-Ann Gwee, ${ }^{2 *}$ Kewin Tien Ho Siah, ${ }^{1}$ Jin Yong Kang, ${ }^{2}$ Rumin Lee ${ }^{3}$ and Cecilia Cheng Lai Ngan ${ }^{4}$ \\ ${ }^{1}$ Department of Gastroenterolgy and Hepatology, National University Health System, Singapore; ${ }^{2}$ Department of Medicine, Yong Loo Lin School \\ of Medicine, National University of Singapore, Singapore; ${ }^{3}$ Stomach, Liver and Bowel Center, Gleneagles Hospital, Singapore; and ${ }^{4}$ Department \\ of Pathology, Singapore General Hospital, Singapore
}

\begin{abstract}
Background/Aims
Non-celiac gluten sensitivity has been increasingly recognized as a predisposing factor for irritable bowel syndrome (IBS)-like symptoms in Western populations where celiac disease (CD) is relatively common. In Asia where CD is rare, we wish to determine the prevalence of gluten protein associated serology in IBS patients, which has not been formally studied, and its relation to histological and human leukocyte antigen (HLA) markers.
\end{abstract}

\section{Methods}

We reviewed a consecutive cohort of Asian patients with IBS, who had undergone serologic testing for IgA against deamidated gliadin peptide antibodies (IgA DGP) and IgA anti-endomysium antibodies, and who also had duodenal biopsies during clinical workup. In addition, a subset of Chinese patients with positive serology was further tested for HLA-DQ2 and HLA-DQ8.

\section{Results}

Of 186 patients, 34 (18\%) were positive for IgA DGP; bloating, abdominal pain, belching and diarrhea were the most commonly reported symptoms but diarrhea as the most bothersome symptom was significantly more common in IgA DGP positive patients. Mildly increased intra-epithelial lymphocytes on duodenal biopsy was also more common (29\% vs. 9\%, $P=0.001)$. Nine of 21 Chinese patients tested as IgA DGP positive undertook HLA-DQ2/DQ8 testing, with only 2 being positive for HLA-DQ8. All patients with positive IgA DGP reported symptom improvement with gluten withdrawal.

\section{Conclusions}

We have described a series of Asian, mainly Chinese, patients with IBS who were tested positive for IgA DGP, and improved on a gluten exclusion diet. We believe this is the first report of non-celiac gluten sensitivity in Asia, a region where CD is uncommon.

(J Neurogastroenterol Motil 2014;20:236-241)

Received: November 15, 2013 Revised: December 27, 2013 Accepted: January 8, 2014

(C) This is an Open Access article distributed under the terms of the Creative Commons Attribution Non-Commercial License (http://creativecommons. org/licenses/by-nc/3.0) which permits unrestricted non-commercial use, distribution, and reproduction in any medium, provided the original work is properly cited.

*Correspondence: Kok-Ann Gwee, FRCP, PhD

Gleneagles Hospital, Annexe Block 05-37, 6A Napier Road, Singapore 258500.

Tel: +65-6474-6848, Fax: +65-6475-8285, E-mail: kok_ann_gwee@nuhs.edu.sg

Financial support: The study was supported by funding from National University Hospital as part of an Academic Development Award.

Conflicts of interest: None.

Author contributions: Wei Lu: study design, data collection and interpretation, and writing the manuscript; Kok-Ann Gwee: concept, study design, Patient recruitment and follow-up, data collection and interpretation, and revising the manuscript; Jin Yong Kang: data interpretation and revising the manuscript; Kewin Tien Ho Siah: data collection and interpretation, and revising the manuscript; Rumin Lee: data collection and revising the manuscript; Cecilia Cheng Lai Ngan: immunology assay interpretation and revising the manuscript. 


\section{Key Words}

Asian; Celiac disease; Gliadin; Gluten sensitive enteropathy; Irritable bowel syndrome

\section{Introduction}

Non-celiac gluten sensitivity (NCGS) relates to one or more of a variety of immunological, morphological or symptomatic manifestations that are precipitated by the ingestion of gluten in people in whom celiac disease (CD) has been excluded. ${ }^{1}$ Although there is no definitive serological marker to distinguish NCGS from other gluten-related disorders, this new entity may suggest a possible link between gluten and irritable bowel syndrome (IBS). ${ }^{2}$ The interest of gluten in IBS patients started more than a decade ago. Sanders et $\mathrm{al}^{3}$ showed that 66 out of 300 (22\%) IBS patients defined by Rome II criteria had positive serology, but the majority of them (43/66) had normal duodenal biopsy. In the same year, Wahnschaffe $\mathrm{U}$ et $\mathrm{al}^{4}$ reported that $30 \%$ had increased celiac antibodies in duodenal aspirate and $23 \%$ with increased intra-epithelial lymphocyte (IEL) counts among 102 IBS patients, but only $9 \%$ having both. In a recent meta-analysis, the prevalence of biopsy-proven CD in patients fulfilling IBS criteria was $4.1 \%$ in a pooled data of 1,464 individuals, ${ }^{5}$ which showed that some patients who initially presented with IBS-like symptoms should have been re-categorized as CD. However, there was a subset of patients without CD who developed gastrointestinal symptoms after gluten ingestion. ${ }^{6}$

Classically CD is diagnosed in a patient with diarrhea and malabsorption, in the presence of intestinal villous atrophy and positivity for gluten related antibodies, who demonstrates rapid clinical and histological response to a gluten free diet. CD is uncommon among Asians, and is particularly rare in people of Chinese ethnic origin. A recent systematic review of celiac disease identified only 18 Chinese patients with biopsy-proven CD. ${ }^{7}$ Studies of Indian populations reported increasing incidence of celiac disease in recent years. ${ }^{8,9}$ One study from UK even showed that a particular Indian clan had a higher incidence of celiac disease than Europeans from the same area. ${ }^{10}$ On the other hand, a few studies from China reported the presence of possible CD among IBS patients, but the incidences were low, and not all of them were histologically confirmed. ${ }^{11,12}$
As far as we know, there are no reports for the other major ethnic groups in Southeast Asia regarding the prevalence of CD or NCGS. We wish to assess the prevalence of anti-deamidated gliadin peptide IgA antibodies (IgA DGP) in Asian patients fulfilling IBS criteria, which may shed some light on the clinical and immunological responses to gluten in this population.

\section{Materials and Methods}

We explored this association by retrospectively studying a cohort of ethnic Asian patients with IBS defined by Rome III criteria, who presented to a single private gastroenterology center in Singapore from year 2007 to 2012. We included all patients of Asian ethnicity who presented with IBS symptoms to the clinic, and excluded those without IBS and without anti-DGP testing as this was a retrospective review. Seventeen non-residents from Indonesia and 21 Indian expatriates working in Singapore were included in this cohort. All patients were tested for IgA DGP and IgA anti-endomysial antibody serology by the indirect immunofluorescence test method (IIFT-Mosaik: Gliadin (GAF-3X)/ Intestine (Monkey), EUROIMMUN). The GAF-3X gliadin used is a repetitive gliadin-analogue fusion peptide consisting of an immunologically dominant deamidated epitope of gliadin with a length of nine amino acids. The patients also underwent gastroscopy, urease test for Helicobacter pylori and duodenal biopsies. Patients who were IgA DGP or IgA anti-endomysium antibody positive were advised to abstain from gluten-containing food. Information on demographics, presenting symptoms and response to gluten-free diet were extracted from case records. In addition, patients of Chinese ethnicity with positive serology were recalled for further testing of human leucocyte antigen (HLA)DQ2 and HLA-DQ8. All data were analysed using statistical software SPSS Statistics Version 10 (SPSS Inc., Chicago, IL, USA).

As this was a retrospective review of patient records, approval was sought and given by the hospital's institution review board (vide PIEC/2013/016) to collate the data in an anonymous method. 


\section{Results}

Of 186 Asian patients included in this study, 34 (18\%) were tested positive for IgA DGP but only one of them was also tested positive for IgA EMA. None of them had diabetes mellitus. Most patients, whether IgA DGP positive or negative, presented with bloating (74\%), abdominal pain (65\%), belching (62\%), diarrhea (47\%), flatulence (44\%) and constipation (29\%) (Table). IgA DGP positive patients were less likely to present with abdominal pain or flatulence compared to IgA DGP negative individuals.

The most bothersome symptoms for IgA DGP positive patients were mainly abdominal pain and diarrhea. There is a trend for IgA DGP positive patients to identify diarrhea as the most bothersome symptoms compared to IgA DGP negative patients, while other symptoms are not significantly different between the 2 groups.

In total, 24 patients (13\%) demonstrated a low grade increase in IEL counts (defined as $>10$ but $<40$ intraepithelial lymphocytes per 100 enterocytes, Marsh 0) in their duodenal biopsies. IgA DGP positive patients were more likely to have increased IEL $(10 / 34=29 \%)$ compared to IgA DGP negative patients $(14 / 152=9 \%, P=0.001)$. Villous blunting (defined as shortened villi without decrease in villous to crypt ratio) was common in IgA DGP positive patients compared to those who were IgA DGP negative, although the difference just failed to reach statistical significance $(15 / 34=44 \%$ versus $42 / 152=27.6 \%, P$ $=0.059)$ (Table). None of these patients had more than Marsh 0 on histology.

Only one patient met the criteria for celiac disease. This was a 26-year-old expatriate from India who presented with bloating associated with loose stools, who had been diagnosed with IBS 2 years ago. There had been no weight loss. Full blood count, erythrocyte sedimentation rate, C-reactive protein, serum vitamin B12, folate and ferritin levels were all normal while IgA DGP and IgA EMA were positive. Duodenal biopsies showed mild intra-epithelial lymphocytosis more than 10 but no greater than 40 per 100 enterocytes, and only villi blunting not amounting to partial villous atrophy. Six months after starting on gluten free diet, his IgA EMA became negative, but IgA DGP remained positive. Nine months later both IgA EMA and IgA DGP were negative, and repeat duodenal biopsies did not reveal any lymphocytosis or villous blunting.

The prevalence of $H$. pylori infection in IgA DGP positive and IgA DGP negative groups was similar, at $17.6 \%$ and $30.9 \%$ respectively. Of the $6 \mathrm{IgA}$ DGP positive patients who were $H$. pylori positive, one had villous blunting and another had increased IEL counts on duodenal biopsy. Of the 47 IgA DGP negative patients who were $H$. pylori positive, 13 had villous blunting, 3 had increased IEL and 4 had both changes on duodenal biopsy. The presence of $H$. pylori did not correlate to in-

Table. Comparison of Demographics, Symptomatology, Endoscopic and Histological Findings Between Anti-deamidated Gliadin Peptide IgA Positive and Negative Irritable Bowel Syndrome Patients

\begin{tabular}{|c|c|c|c|}
\hline & $\begin{array}{c}\operatorname{IgA} \operatorname{DGP}(+) \\
(\mathrm{n}=34)\end{array}$ & $\begin{array}{l}\operatorname{IgA} \operatorname{DGP}(-) \\
\quad(\mathrm{n}=152)\end{array}$ & $P$-value \\
\hline Age (mean $[\mathrm{SD}], \mathrm{yr})$ & $42.5(10.8)$ & $43.0(12.9)$ & 0.589 \\
\hline Female (n $[\%])$ & $24(71)$ & $75(49)$ & \multirow{2}{*}{0.025} \\
\hline Male:Female & $10: 24$ & $77: 75$ & \\
\hline \multicolumn{4}{|c|}{ Prevalence of IgA DGP by ethnic group (n [\%]) } \\
\hline Chinese $(\mathrm{n}=124)$ & $21(17)$ & $103(83)$ & 0.548 \\
\hline Indonesian $(\mathrm{n}=17)$ & $7(41)$ & $10(59)$ & 0.018 \\
\hline Indian $(\mathrm{n}=37)$ & $5(14)$ & $32(86)$ & 0.483 \\
\hline Eurasian $(\mathrm{n}=5)$ & $1(20)$ & $4(80)$ & 1.000 \\
\hline Others $(\mathrm{n}=3)$ & $0(0)$ & $3(100)^{\mathrm{a}}$ & - \\
\hline \multicolumn{4}{|c|}{ Symptoms at presentation (n $[\%])$} \\
\hline Abdominal pain & $22(65)$ & $125(82)$ & 0.023 \\
\hline Bloating & $25(74)$ & $125(82)$ & 0.245 \\
\hline Constipation & $10(29)$ & $45(30)$ & 0.982 \\
\hline Diarrhea & $16(47)$ & $70(46)$ & 0.915 \\
\hline Belching & $21(62)$ & $85(56)$ & 0.534 \\
\hline Flatulence & $15(44)$ & $107(70)$ & 0.004 \\
\hline Vomiting & $1(3)$ & $2(1)$ & 0.496 \\
\hline \multicolumn{4}{|c|}{ Most bothersome symptom (n [\%]) } \\
\hline Abdominal pain & $10(29)$ & $62(41)$ & 0.25 \\
\hline Bloating & $7(21)$ & $46(30)$ & 0.30 \\
\hline Constipation & $2(6)$ & $7(5)$ & 0.67 \\
\hline Diarrhea & $10(29)$ & $19(12)$ & 0.02 \\
\hline Belching & $2(6)$ & $7(5)$ & 0.67 \\
\hline Flatulence & $2(6)$ & $8(5)$ & 1.00 \\
\hline Other symptoms & $1(3)^{b}$ & $3(2)^{c}$ & NA \\
\hline \multicolumn{4}{|c|}{ Endoscopic and histological findings (n $[\%]$ ) } \\
\hline H. pylori $(+)$ & $6(18)$ & $47(31)$ & 0.112 \\
\hline Villous blunting & $15(44)$ & $42(28)$ & 0.059 \\
\hline IEL & $10(29)$ & $14(9)$ & 0.001 \\
\hline
\end{tabular}

${ }^{\mathrm{a}}$ These 3 patients are Japanese, Korean and Vietnamese, respectively; ${ }^{\mathrm{b}}$ Choking

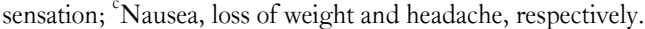

IgA DGP, anti-deamidated gliadin peptide IgA antibodies; IEL, intra-epithelial lymphocytosis; NA, not applicable.

Villous blunting $=$ villous to crypt ratio of $<3: 1$. Intra-epithelial lymphocytosis $=$ more than 10 lymphocytes per 100 enterocytes.

All $P$-values were calculated using Fisher's exact test. 
creased IEL counts $(P=0.468)$ in either group.

There were 124 Chinese patients including 8 Indonesian Chinese in this cohort. Their demographics were not different from the other patients. Twenty-one (17\%) were positive for IgA DGP. Among 9 of the $21 \mathrm{IgA}$ DGP positive Chinese patients who underwent HLA-DQ2/DQ8 genotyping, 2 patients were tested positive for HLA-DQ8. These 2 patients were sisters whose parents had emigrated from northern China where the traditional dietary staple was wheat. Neither of them had villous blunting, but one had increased IEL. The ancestry of the other 7 patients who were HLA-DQ2/DQ8 genotype negative was traced to southern China where the traditional staple diet is rice; one of the patients had concurrent systemic lupus erythematosus. Five of these 7 patients had villous blunting, and 2 had increased IEL.

\section{Discussion}

$\mathrm{CD}$ was rarely reported in non-Indian Asian populations and gluten related disorders have not to date been recognized as a significant clinical problem. ${ }^{11,12}$ While classical $\mathrm{CD}$ is diagnosed on the basis of typical symptoms of malabsorption, positive serology with histological findings of villous atrophy or IEL > 40/100 enterocytes, ${ }^{1}$ the new entity of non-celiac gluten sensitivity has recently been described. Recent consensus proposed that definitions of NCGS are cases with negative anti-EMA and/or anti-tissue transglutaminase ( $\mathrm{tTG}$ ), normal duodenal histopathology and with the possible presence of biomarkers of native gluten immune-reaction such as anti-gliadin antibodies (AGA). ${ }^{13}$

Gliadin is a component of gluten, which plays an important part in the immunogenicity of gluten in CD. The dominant epitope responsible for the T-cell response appeared to be a deamidated glutamine residue of gliadin. Gluten refers to the complex of water insoluble proteins from wheat, rye and barley. It is poorly digested in the human intestine with or without $\mathrm{CD}$, and crosses intactly into the submucosa of the small intestine. The gluten peptide is then deamidated in the submucosa by tTG. Deamidation enhances binding to HLA-DQ2 and HLA-DQ8 in susceptible individuals, and subsequently triggers inflammatory responses. ${ }^{1,14-15}$ Gliadin also contains peptides that are able to activate an innate immune response, in particular, inducing a selective expansion of IEL. ${ }^{16}$ More IgA DGP positive patients had increased IEL counts $(P=0.001)$. We speculate that DGP provokes a milder form of inflammatory process in our study population, with normal or minimal changes in enterocyte architecture and less pro- nounced IEL. This inflammatory process then manifests clinically as IBS symptoms predominantly with diarrhea, which was identified as most bothersome symptom in IgA DGP positive IBS patients.

The original IgA AGA test has a low diagnostic accuracy and is therefore no longer recommended for initial diagnostic screening for $\mathrm{CD} .{ }^{17}$ The new synthetic IgA DGP used in our study has a higher sensitivity and is comparable to IgA anti-tTG. ${ }^{18}$ Indeed, a recent study has shown that the sensitivity of IgA DGP was superior to anti-tTG and comparable to EMA in early-stage celiac disease with normal villous morphology. ${ }^{19}$ On the other hand, IgG AGA was present in more than half of patients with NCGS, with a few patients having IgA AGA or IgG DGP ${ }^{20}{ }^{20}$ In the same study, there was good congruence among IgG DGP, IgA EMA and $\operatorname{IgA}$ tTG for $\mathrm{CD}$. This is similar to the findings reported by Adler et $\mathrm{al}^{21}$ and Carroccio et al. ${ }^{22}$ Close to half of their patients with gluten sensitivity were positive for either IgG and/or IgA AGA, but only a few of them were EMA positive, showing only mild changes on wireless capsule endoscopy, and these patients were deemed not to have $\mathrm{CD}^{21,22}$ Eighteen percent of our patients were IgA DGP positive among whom all but one were IgA EMA negative, and less than half had villous blunting. In addition, one of our patients with positive IgA DGP was diagnosed with systemic lupus erythematosus, suggesting an autoimmune diathesis. More studies are needed to explore the significance of isolated IgA DGP positivity in NCGS, and determine whether it is a transient immune response to gluten challenge.

The prevalence of HLA-DQ2 and HLA-DQ8 in Southeast Asia is unclear. The frequency of HLA-DQ2 is high in northern China along the Silk Route, where the consumption of wheat is higher than in the south. In Jiangsu Province, along Yangtze River, the frequency of HLA-DQ2 is $7.2 \%$ and the frequency of HLA-DQ8 is $4.7 \%{ }^{23,24}$ There is an association of HLA-DQ2 with gluten sensitivity, although this is weaker than its linkage with CD. HLA-DQ2 in NCGS may be related to presence of biomarkers of innate gluten reaction such as AGA. It has been suggested that this newly recognized and incompletely understood entity of gluten sensitivity is part of the spectrum of gluten related disorders. ${ }^{3,25}$ In a prospective gluten challenge study, the prevalence of HLA-DQ2 or DQ8 was 53\% in the NCGS group and $100 \%$ in the CD group. ${ }^{21}$ The low prevalence of HLA-DQ8 and absence of HLA-DQ2 in our patients confirm that our population has a low genetic susceptibility to $\mathrm{CD}$, yet there is significant gluten sensitivity, suggesting that dietary rather than genetic factors are relevant in the emergence of NCGS in this part 
of the world.

Increased IEL can be a non-specific finding associated with many non-CD causes such as drugs, infection, immune dysregulation, inflammatory bowel disease, microscopic colitis, sarcoidosis and IgA deficiency. It was shown that there may be an association with IBS in those cases with no apparent causes identified, and the IEL count becomes normal on repeat biopsy in $76 \%{ }^{26}$ In our study, histopathology findings were close to normal with findings of only villous blunting and increased IEL of more than 10 but less than 40 enterocytes - no more that Marsh Grade 0, suggesting the possibility of immune responses to gluten not amounting to $\mathrm{CD}$. The presence of $H$. pylori did not appear to affect the IEL counts in both IgA DGP positive and negative groups.

Our study has a few limitations. Firstly, only IgA DGP and IgA EMA but not IgA tTG, were tested. Future studies may include all three serologic markers to compare their sensitivity and specificity in diagnosing NCGS. Furthermore only patients with positive IgA DGP were advised to go on GFD so the response to GFD in IgA DGP negative cases is not known. As our study is a retrospective review of clinical notes, detailed health history and food gradient were not available. Lastly, no repeat serological tests and duodenal biopsies were done to assess response to GFD, and to determine whether the positive serology is a transient immune response to gluten.

In conclusion, we have described a series of Asian, mainly Chinese, patients with IBS by Rome III criteria who have serological markers and changes in duodenal biopsies that would be consistent with non-celiac gluten sensitivity. This is novel as this entity has not to our knowledge been reported in the Asian literature. As this was a clinical observation study, prospective studies are required to verify the association of IBS with NCGS in Asian populations.

\section{References}

1. Ludvigsson JF, Leffler DA, Bai JC, et al. The Oslo definition for celiac disease and related terms. Gut 2013;62:43-52.

2. Vertu EF. Editorial: Can gluten contribute to irritable bowel syndrome? Am J Gastroenterol 2011;106:516-518.

3. Sanders DS, Carter MJ, Hurlstone DP, et al. Association of adult coeliac disease with irritable bowel syndrome: a case-control study in patients fulfilling ROME II criteria referred to secondary care. Lancet 2001;358:1504-1508.

4. Wahnschaffe U, Ullrich R, Riecken EO, Schulzke JD. Celiac disease-like abnormalities in a subgroup of patients with irritable bowel syndrome. Gastroenterology 2001;121:1329-1338.
5. Ford AC, Chey WD, Talley NJ, Malhotra A, Spiegel BM, Moayyedi P. Yield of diagnostic tests for celiac disease in individuals with symptoms suggestive of irritable bowel syndrome: systematic review and meta-analysis. Arch Intern Med 2009;169:651-658.

6. Biesiekierski JR, Newnham ED, Irving PM, et al. Gluten causes gastrointestinal symptoms in subjects without celiac disease: a double-blind randomized placebo-controlled trial. Am J Gastroenterol 2011;106:508-514.

7. Kang JY, Kang AH, Green A, Gwee KA, Ho KY. Systematic review: worldwide variation in the frequency of coeliac disease and changes over time. Aliment Pharmacol Ther 2013;38:226-245.

8. Sood A, Midha V, Sood N, Kaushal V, Puri H. Increasing incidence of celiac disease in India. Am J Gastroenterol 2001;96:2804-2805.

9. Dutta AK. Chacko A, Avinash B. Suboptimal performance of IgG anti-tissue transglutaminase in the diagnosis of celiac disease in a tropical country. Dig Dis Sci 2010;55:698-702.

10. Sher K, Fraser RC, Wicks AC, Mayberry JF. High risk of coeliac disease in Punjabis. Epidemiological study in the south Asian and European populations of Leicestershire. Digestion 1993;54:178-182.

11. Jiang LL, Zhang BL, Liu YS. Is adult celiac disease really uncommon in Chinese? J Zhejiang Univ Sci B 2009;10:168-171.

12. Wu J, Xia B, von Blomberg BM, et al. Coeliac disease: emerging in China? Gut 2010;59:418-419.

13. Sapone A, Bai JC, Ciacci C, et al. Spectrum of gluten-related disorders: consensus on new nomenclature and classification. BMC Medicine 2012;10:13

14. Anderson RP, Degano P, Godkin AJ, Jewell DP, Hill AV. In vivo antigen challenge in celiac disease identifies a single transglutaminase-modified peptide as the dominant A-gliadin T-cell epitope. Nat Med 2000;6:337-342.

15. van de Wal Y, Kooy Y, van Veelen P, et al. Selective deamidation by tissue transglutaminase strongly enhances gliadin-specific $\mathrm{T}$ cell reactivity. J Immunol 1998;161:1585-1588.

16. Gianfrani C, Auricchio S, Troncone R. Adaptive and innate immune responses in celiac disease. Immunol Lett 2005;99:141-145.

17. James SP. National Institutes of Health Consensus Development Conference Statement. Celiac Disease June 28-30, 2004. Gastroenterology 2005;128(suppl 1):S1-S9.

18. Niveloni S, Sugai E, Cabanne A, et al. Antibodies against synthetic deamidated gliadin peptides as predictors of celiac disease: prospective assessment in an adult population with a high pretest probability of disease. Clin Chem 2007;53:2186-2192.

19. Kurppa K , Lindfors $\mathrm{K}$, Collin $\mathrm{P}$, et al. Antibodies against deamidated gliadin peptides in early-stage celiac disease. J Clin Gastroenterol 2011;45:673-678.

20. Volta U, Tovoli F, Cicola R, at el. Serological tests in gluten sensitivity (nonceliac gluten intolerance). J Clin Gastroenterol 2012;46: 680-685.

21. Adler SN, Jacob H, Lijovetzky G, Mulder CJ, Zwiers A. Positive coeliac serology in irritable bowel syndrome patients with normal duodenal biopsies:video capsule endoscopy findings and HLA-DQ typing may affect clinical management. J Gastrointestin Liver Dis 2006;15:221-225.

22. Carroccio A, Mansueto P, Iacono G, et al. Non-celiac wheat sensitivity diagnosed by double-blind placebo-controlled challenge: exploring a new clinical entity. Am J Gastroenterol 2012;107:1898-1906. 
23. Yu RB, Hong X, Ding WL, Tan YF, Wu GL. Polymorphism of the HLA-DQA1 and -DQB1 genes of Han population in Jiangsu Province, China. Chin Med J 2006;119:1930-1933.

24. Wu J, Xia B, von Blomberg BM, et al. Coeliac disease in China, a field waiting for exploration. Rev Esp Enferm Dig 2010;102:472-477.

25. Ferch CC, Chey WD. Irritable bowel syndrome and gluten sensi- tivity without celiac disease: separating the wheat from the chaff. Gastroenterology 2012;142:664-666.

26. Aziz I, Evans KE, Hopper AD, Smillie DM, Sanders DS. A prospective study into the aetiology of lymphocytic duodenosis. Aliment Pharmacol Ther 2010;32:1392-1397. 